\title{
Acute gastroenteritis in children of the world: what needs to be done?
}

Position paper of the Federation of International Societies of International Societies of Pediatric Gastroenterology, Hepatology and Nutrition (www.FISPGHAN.org)

A. Guarino ${ }^{1}$, J. Aguilar ${ }^{2}$, J.A. Berkley ${ }^{3}$, I. Broekaert ${ }^{4}$, R.V. Vazquez-Frias ${ }^{5}$, L.R. Holtz ${ }^{6}$, A. Lo Vecchio $^{1}$, T. Meskini ${ }^{7}$, S.R. Moore ${ }^{8}$, J. Rivera ${ }^{9}$, B. Sandhu ${ }^{10}$, A. Smarrazzo ${ }^{1}$, H. Szajewska ${ }^{11}$, S. Treepongkaruna ${ }^{12}$

${ }^{1}$ Department of Translational Medical Science, Section of Pediatrics, University of Naples Federico II. Coordinator of FISPGHAN working group on acute gastroenteritis (ESPGHAN) ${ }^{2}$ Department of Pediatrics, University of the Philippines Manila - Philippine General Hospital; Institute of Pediatrics and Child Health, St. Luke's Medical Center, Quezon City, Philippines (APPSGHAN)

${ }^{3}$ KEMRI/Wellcome Trust Research Programme, PO Box 230-80108, Kilifi, Kenya \& Centre for Tropical Medicine \& Global Health, University of Oxford, UK (CAPGAN)

${ }^{4}$ University Children's Hospital, Cologne, Germany (ESPGHAN)

${ }^{5}$ Hospital Infantil de México Federico Gomez, Mexico City, Mexico; Latin Aamerican Society for Pediatric Gastroenterology, Hepatology and Nutrition (LASPGHAN)

${ }^{6}$ Department of Pediatrics, Washington University School of Medicine, St. Louis, MO USA 63110 (NASPGHAN)

${ }^{7}$ Faculty of Medicine and pharmacy of Rabat. Mohammed V University of Rabat- Rabat Children's Hospital. Rabat (Morocco) (PASPGHAN) ${ }^{8}$ Division of Pediatric Gastroenterology, 
Hepatology, and Nutrition Department of Pediatrics University of Virginia Charlottesville. (NASPGHAN)

${ }^{9}$ Universidad Nacional Mayor de San Marcos and Universidad Peruana Cayetano Heredia Hospital del Niño; National Institute of Pediatrics, Lima- Peru (LASPGHAN)

${ }^{10}$ Department of Paediatric Gastroenterology and Nutrition, Bristol Royal Hospital for Children, Bristol BS2 8BJ. UK. (CAPGAN)

${ }^{11}$ Department of Paediatrics, The Medical University of Warsaw, Warsaw, Poland. (ESPGHAN)

${ }^{12}$ Department of Pediatrics, Faculty of Medicine Ramathibodi Hospital, Mahidol University, Bangkok, Thailand (APPSGHAN)

Correspondence: Alfredo Guarino. Department of Translational Medical Science, Section of Pediatrics. University of Naples Federico II, Via S. Pansini 5, 80131 Naples 


\section{Abstract}

The incidence of gastroenteritis has greatly reduced due to improved hygiene conditions in developing countries and the use of Rotavirus vaccine. However, still thousands of children die from gastroenteritis, most of them in poor countries. Yet gastroenteritis management is simple, inexpensive, and effective and is largely the same all over the world. Universal guidelines for gastroenteritis guide the management and include simple interventions put forward early in the course of the disease. Treatment includes rehydration, continuing oral feeding, and antiinfective drugs in selected clinical conditions related to the symptoms or to host-related risk, and possible additional drug treatment to reduce the duration and severity of symptoms. There may be minor geographical differences in the treatment applied due to health care organizations that do not substantially change the standard universal recommendations. Prevention is recommended with sanitation interventions and Rotavirus universal immunization. Implementation of those interventions through educational initiatives and local programs in target areas are needed. A series of recommendations for interventions, education, and research priorities are included here with the aim of reducing the burden of gastroenteritis, to be pursued by scientists, physicians, policy makers, and stakeholders involved. They include the need of recommendations for the management of gastroenteritis in malnourished children, in those with chronic conditions, in neonates, and in emergency settings. A reference system to score dehydration, the definition of optimal composition of rehydration solution and the indications for anti-infective therapy are also included. Rotavirus immunization should be actively promoted, and evidence-based guidelines should be universally implemented. Research priorities are also indicated.

Keywords: Gastroenteritis; Rotavirus; implementation; prevention

\section{What is known}


- Gastroenteritis is still a major cause of death in children and of costs for societies and families worldwide.

- In addition, its epidemiology and other clinical and experimental data open now threats and new hopes for prevention and management

\section{What is new}

- This position paper provides a list of priorities that require attention and resources.

- Among the others, the composition of oral rehydration solution should be revised, antiinfective therapy should be better standardized, and guidelines should be reviewed and implemented.

- Research on pathogens and their mechanisms and new drugs is needed.

- Finally, at risk groups including malnourished children should be the target for prevention and management initiatives. 


\section{Brief summary of the problem and FISPGHAN activities}

Acute gastroenteritis (AGE) is a common cause of morbidity and mortality in infants and children. A child younger than 5 years may experience as many as 1 to 5 episodes of acute diarrhea each year. According to the Global Health Data Exchange in 2016, diarrhea was the $8^{\text {th }}$ leading cause of death among all ages ( 1.65 million deaths) and the $5^{\text {th }}$ leading cause of death among children below 5 years (446 000 deaths). Malnutrition, unsafe water, and sanitation continue to be leading risk factors for diarrhea. ${ }^{1}$ Almost $40 \%$ of these deaths have been putatively linked with Rotavirus (RV) and most occur in low-income countries. However, AGE is also an important health problem in developed countries as it leads to frequent emergency room visits, hospital admissions, and high costs to both society and families, and death is not exceptional (in western European countries approximately 300 children die annually due to gastroenteritis).

If AGE and dehydration are recognized and treated promptly and appropriately, the prognosis of diarrhea is good. Therapy is crucial, easy, and inexpensive. The major goal is to restore hydration and prevent dehydration, which can be achieved with oral rehydration solution (ORS). Avoiding fasting and continuing feeding are also important. In striking contrast, in rich countries and in private facilities in poor countries, unnecessary medical interventions are often applied, including inappropriate hospital admissions, microbiological investigations, diet restrictions, and medication administration.

In terms of prevention, hygiene measures (particularly the use of clean water and toilet systems) that can successfully reduce fecal contamination, and immunization particularly against $R V$, strongly reduce morbidity and mortality. Developing and applying the means of effective education of mothers in the early recognition of acute diarrhea as a potentially dangerous condition and what actions to take, is extremely important particularly in developing countries. 
The Federation of Pediatric Societies of Gastroenterology Hepatology and Nutrition (FISPGHAN) has prioritized AGE since its foundation. In 2012, a FISPGHAN expert working group (WG) identified priorities for medical intervention, education, and research to reduce the morbidity and mortality associated with AGE, which are still valid ${ }^{2}$ (Table 1). Some have been achieved, also with the active intervention by FISPGHAN. In addition to indicating the priorities, the WG directly promoted important initiatives. A paper that described a comparison of the recommendations for the management of gastroenteritis using available guidelines demonstrate major similarities ${ }^{3}$. Based on this paper, the WG formulated universal recommendations for the management of acute diarrhea ${ }^{4}$, took an active role in education initiatives in developing countries, and tested the efficacy of e-learning programs ${ }^{5}$. Additionally, two informative videos were prepared by a group of young pediatric gastroenterologists targeting caretakers and social workers explaining the clinical signs, alarming conditions, and principles of management of gastroenteritis (https://www.youtube.com/watch?v=IYW9hpcAKwk). These videos have been translated in different languages.

In this report, a group of experts from all the societies forming FISPGHAN provide a global summary of AGE and indicate the major interventions needed to improve the health condition of children with a particular focus on low-income countries, which is the ultimate mission of FISPGHAN. Moreover, a list of unresolved problems, which should be the focus of future research and endeavors, is also included.

The paper was not intended to produce recommendations on how to treat or prevent gastroenteritis. Rather it aims at briefly providing a picture of the current situation and open issues and provide a list of priorities for improving management and prevention. Priorities include recommendations for management, research issues and further initiatives that FISPGHAN could engage or promote. 
The paper was constructed by agreeing on a list of topics that were handled either directly by the members appointed by the societies forming FISPGHAN or by outer experts that are included as authors. An extensive discussion took place among members which eventually led to the list of recommendations that are included in the last part of the paper and reflect the position of the societies forming FISPGHAN about what needs to be done by political, scientific and health care agencies and institutions, (including FISPGHAN) to reduce the burden of gastroenteritis worldwide.

\section{Changes and novelties in the epidemiology of acute diarrhea}

Launched in the year 2000, the Millennium Development Goal 4 (MDG4), had the ambitious aim of reducing the global mortality rate of children under 5 years by 2015 by two-thirds. MDG4 was aimed at reducing childhood mortality due to acute diarrhea ${ }^{6}$. However, the goal was not fully achieved, and substantial challenges remain to be addressed.

In 2015, the MDGs were replaced by the more comprehensive, although less health-focused, Sustainable Development Goals (SDGs). SDG 3.2 is the main indicator for improving child survival, with the aim of reducing mortality of children below the age of 5 years to fewer than 25 deaths per 1000 live births, decreasing neonatal mortality to fewer than 12 deaths per 1000 live births, and eradicating preventable deaths of newborns and children younger than 5 years by $2030^{7}$.

In 2015, mortality due to diarrheal diseases and lower respiratory infections decreased in children below the age of 5 years due to the continued expansion of routine immunization programs, improved sanitation, and better access to health services and care-seeking behaviors for childhood illnesses, particularly in lower-income countries. However, diarrheal diseases remain an important health problem globally and the results of MDG4 reduction have not been consistent across all regions, with Sub-Sahara Africa and South Asia reporting considerable mortality. 
Viruses are the most common agents of AGE, accounting for more than $60 \%$ of pediatric cases, followed by bacteria and parasites ${ }^{8-10}$. The prevalence of individual pathogens varies widely between geographic areas and different age groups but globally RV infection remains the leading cause of diarrhea in children less than 5 years old ${ }^{10,11}$.

Molecular methods have substantially increased the sensitivity of pathogen detection and have expanded the understanding of etiologic agents in diarrhea ${ }^{10-12}$. In a large cohort of children between 0 and 2 years with AGE, quantitative polymerase chain reaction (PCR) identified pathogens in $65 \%$ of episodes, compared to $33 \%$ using traditional microbiologic studies ${ }^{12}$. Thus, some pathogens such as heat-stable enterotoxin producing Escherichia coli (ST-ETEC), Shigella, and Cryptosporidium were previously underestimated ${ }^{13}$. These pathogens are common agents of moderate to severe diarrhea in young children in low- resource countries ${ }^{9}$. Quantitative PCR determinations supported higher estimates of these pathogens and increased the attributable incidence for Shigella and Campylobacter jejuni/coli twofold, for ST-ETEC 1.5-fold, and for adenovirus 40/41 five-fold, compared with estimates based on culture or immunoassay $^{11}$.

Interestingly, enterotoxin-producing $E$. coli was found to be a frequent agent of mild to moderate gastroenteritis also in high income countries ${ }^{13}$, raising the hypothesis that there may have differences in severity rather than the issue of distribution between low- and high- income countries.

Currently, several multiplex nucleic acid tests are available for the detection of a spectrum of enteric pathogens ${ }^{14}$. The advantages of these tests include the breadth of pathogens discovered, the rapid turnaround, and the accuracy of their results. However, the challenges and setbacks include the high costs, limitations in identifying the true causative role of multiple pathogens in a stool sample of an affected patient, and the lack of bacterial isolates needed for antibiotic susceptibility and outbreak analysis. At present, the understanding of the prognostic, 
therapeutic, and pathogenic significance of multiple agents in a stool sample is limited, making it difficult for the clinician to evaluate the results of these special tests.

\section{Vaccines for prevention of acute diarrhea}

Various enteric vaccines have been developed to reduce the incidence of acute diarrhea including cholera, typhoid, and RV vaccines ${ }^{15-17}$. Because the efficacy rates of the currently available oral killed whole cell cholera vaccine are between $50 \%$ and $60 \%$, its inclusion in national immunization programs of endemic countries will depend on the prevalence of cholera, the frequency of outbreaks, and accessibility of healthcare services ${ }^{15}$. A similar approach applies to typhoid vaccination. Thus, until further developments, wide-scale implementation of typhoid vaccinations will be evaluated in the light of efficacy rates and the burden of the disease in a particular country or setting ${ }^{16}$.

\section{Efficacy of vaccines and their differences in high-and low-income areas}

$\mathrm{RV}$ vaccine plays a major role in preventing $\mathrm{AGE}^{18}$. The two commercially available $\mathrm{RV}$ vaccines, prequalified by the $\mathrm{WHO}$, are the human monovalent vaccine and human-bovine reassortment pentavalent vaccine. Other two monovalent vaccines have been developed and made available in India and China. Differences in efficacy rates have been noticeably evident for high- and low-income countries [Table 2]. The efficacy of RV vaccines is lower in lowincome countries. The reasons are still unclear, however, differences in gut microbiome and HLA groups, titer of anti-RV IgA in mother's breast milk, as well as a possible interference of oral polio-vaccine, might play a role in determining a lower efficacy in these settings. However, due to the higher burden of diarrhea in these countries, more cases can be prevented in these areas. No increased risk of serious adverse events has been observed for any RV vaccines in both the high- and low-income countries.

\section{Dissemination and barriers to immunization}


The efficacy of RV vaccines has been well established. A decade following the global dissemination, RV immunization has been included in the national and subnational immunization programs of 92 countries. A reduction of $>80 \%$ and $50 \%$ in disease burden has since been observed in both high- and low-income countries, respectively ${ }^{18}$. Recently, in a few European countries, targeted RV immunization has been proposed, e.g., for preterm infants only, but the general conclusion is that RV immunization should be universally offered to all infants regardless of geography, age, and health condition ${ }^{19}$. (European Academy of Paediatrics and ESPID, under review)

The FISPGHAN WG on AGE conducted a specific study on the barriers limiting RV immunization worldwide ${ }^{20}$. The single most frequent barrier in this study was the cost. However, the lack of perception of severity of AGE is also a significant barrier ${ }^{20}$ [Fig. 1]. Another major factor for the limited implementation of RV vaccination in countries with high disease burden remains the logistical support for the program. Failure to grasp the ease of disease transmission with a low viral load is another issue.

Several new vaccines are in the pipeline. As there are no licensed vaccines against enterotoxigenic Escherichia coli (ETEC), the development of a suitable candidate vaccine could be beneficial for children in low-income countries and for travelers. Currently, an attenuated ETEC vaccine candidate, ACE527, that is co-administered with a mucosal adjuvant the doublemutant heat-labile toxin (dmLT) of ETEC, has demonstrated a protective efficacy rate of $66 \%$ against severe diarrhea among human volunteers challenged with virulent ETEC strain H10407 21.

\section{Comparison of recommendations in different countries, development of universal recommendations for non-malnourished children and main novelties about management of acute diarrhea.}


Clinical practice guidelines universally recommend rapid assessment of dehydration and shock. Although most guidelines recommend that the actual loss of body weight be used to quantitatively estimate dehydration ${ }^{4}$, prior data on the weight may be unavailable. Scoring systems based on clinical signs are suggested by the WHO and other institutions, although their reliability may be limited to selected settings ${ }^{22}$. The Clinical Dehydration Scale is an easy tool supported by guidelines and is reliable in estimating diarrhea induced fluid losses ${ }^{22}$. Mild to moderate uncomplicated AGE without underlying comorbidity or dysentery (as indicated by bloody stools) does not require routine microbiological or other laboratory work- up ${ }^{4}$.

The management of AGE is based on 4 pillars: 1) rehydration; 2) anti-infective therapy; 3) additional treatment of AGE/diarrhea to reduce its duration and severity; and 4) nutritional management:

1) Rehydration. The primary objective of management of diarrhea is to prevent potentially fatal complications including dehydration, metabolic acidosis, electrolyte disturbances, and sepsis. Achieving rehydration relies on the administration of rehydration solution via the oral or, in a small proportion of carefully selected children, the intravenous route. Most clinical guidelines recommend low osmolarity $\left[\leq 75 \mathrm{mmol} / \mathrm{L} \mathrm{Na}^{+}\right] \mathrm{ORS}^{3}$, but lower concentrations of $\mathrm{Na}^{+}$have been used successfully. Thus, the optimal composition of ORS is debatable. The advantage of having a single ORS is quite clear and WHO ORS has been the most important preparation achieved by WHO since its formulation. On the other hand, the knowledge, epidemiology, and management of AGE have changed through the years and it may be timely to reconsider the composition of ORS taking into account efficacy, costs, stability, and palatability.

Although palatability may be an issue in children with no or mild dehydration, $\mathrm{Na}^{+}$ concentration and its stoichiometric ratio with glucose should be optimized based on intestinal fluid absorption. Vomiting may impair oral rehydration. The use of ondansetron, particularly in 
emergency settings, may prove useful in overcoming a dangerous phase of AGE, and prevent hospital admission or the need of intravenous rehydration. This has been shown in interventions of improved clinical practice and in high-income and low-income countries ${ }^{24}$. Nasogastric administration of ORS, although not widely used as a second-line treatment, is an effective alternative to intravenous fluids particularly in resource-poor settings ${ }^{25}$.

2) Anti-infective therapy is still an unresolved issue in acute gastroenteritis ${ }^{26}$. There are two potential reasons for deciding on the use of antibiotic therapy in gastroenteritis: clinical conditions and host-related risk factors. Microbiological investigation is generally not indicated in AGE, unless anti-infective therapy is considered. Most guidelines recommend not giving antibiotics unless there is sepsis, cholera, or significant comorbidity. Children with suspected or confirmed invasive disease or with sepsis, demonstrated by blood culture or by an increase in inflammation markers, including C-reactive protein or neutrophilic leukocytosis should receive antibiotics once fecal samples have been obtained. In absence of sepsis, or immunosuppression, antibiotics should be used in response to culture results, or endemic infections specific to region of the world. "Dysentery", and inflammation with elevated markers, do not always warrant antibiotic treatment, depending on the pathogen.

An example may be the isolation of Shiga toxin- producing E. coli (E coli 0157:H7) in the stools, in that case the use of antibiotics in absence of sepsis or underlying conditions is contraindicated. The potential indications and choice of antibiotics for anti-infective therapy are listed in Table 3. Hence the potential reasons for requiring a microbiological investigation of stools correspond to potential indications for anti-infective therapy, although the criteria for testing and treatment are difficult to develop. The etiology affects therapy and there is evidence of significant benefit for the use of antibiotics in diarrhea caused by Vibrio cholerae, and less strong evidence for Shigella and for persistent diarrhea. ${ }^{27-29}$ Children infected with Shiga toxin-producing $E$. coli should not receive antibiotics. If $C$. difficile is 
obtained from an otherwise healthy child with AGE, it should not be treated. For nontyphoidal Salmonellae, there are no universal recommendations and it is suggested to treat with antibiotics all infants $<3-6$ months of age due to an increased risk of invasive disease $\mathrm{e}^{30}$.

Severely immunocompromised children should be tested and, even if negative, considered for empirical antimicrobial treatment. Severe RV diarrhea in the hospital setting may be effectively treated with oral administration of human immunoglobulins given once at 400 $\mathrm{mg} / \mathrm{kg}$ body weight ${ }^{31}$. Severe acute malnutrition is also an indication for antibiotic therapy, independent of the presence of diarrhea ${ }^{32}$. The choice of antibiotics and duration of treatment are far from being standardized. However, based on etiology and risks factors, the most common indications are listed in table 3B.

3) Additional treatment of diarrhea aims at reducing the duration and severity of symptoms and encompasses a number of heterogeneous therapies. Oral administration of zinc and early refeeding with an age-appropriate diet, are recommended in all clinical guidelines ${ }^{3}$. In countries where many children are at risk of malnutrition, zinc is part of routine management of AGE. Zinc should be given as a therapy (usually tablet of $20 \mathrm{mg} /$ day).

Several interventions have been proposed with the aim of reducing the duration and severity of symptoms. However, they should always be considered as adjunct to rehydration and based on solid evidence. There is a discussion about the efficacy and importance of interventions put forward to reduce the duration and intensity of AGE. There is a fear that these interventions may detract from rehydration which is the basic therapy of AGE. In addition, not all interventions proposed as adjunct to oral rehydration, are effective nor are they necessarily a priority in terms of public health concern, particularly under resourcerestricted conditions. On the other hand, the perspective of shortening the symptoms of an individual child and his/her family should not be neglected and if there is an affordable and effective intervention to shorten diarrhea even by one day, the intervention may be 
considered. The features of the ideal drug for AGE are reported in table 4. Based on current available data the physician may select effective intervention to be administered to shorten the duration of symptoms.

4) Nutritional management. Nutrition is an important component in the management of AGE. The current recommendation is to continue feeding the child with AGE with a regular diet without restrictions. The nutritional approach however is considerably affected by local traditions and several dietary modifications are applied in many geographical locations, generally with little or no evidence of efficacy. The efficacy of a lactose-free diet in hospitalized children with AGE has been shown in a meta-analysis of 33 trials, of which 28 were performed in an inpatient setting. Lactose-free diet was associated with a shorter duration of diarrhea and reduced treatment failures. It may be also effective if diarrhea persist over $7 / 14$ days. The data however did not include malnourished children nor were they performed in countries where mortality is high ${ }^{33} \mathrm{~A}$ trial of lactose-free feeding in malnourished children showed no impact on diarrhea ${ }^{34}$. In conclusion, the management of AGE is simple as shown in the algorithm reported in Figure $2^{35}$.

\section{Paucity of evidence in the management of diarrhea in malnourished or at-risk or immunocompromised children.}

Diarrhea was the second leading cause of death in low-income countries in 2016, and most of these cases were children with severe malnutrition. AGE is a strong predictor of mortality in malnourished children ${ }^{36,37}$. The treatment of AGE in the malnourished child is part of the updated WHO guideline on the management of severe acute malnutrition in infants and children $^{38}$. The evidence for the recommendations was low or very low, due to the limited availability of randomized controlled trials comparing existing WHO recommendations with new treatment options. Where direct evidence was not available, indirect evidence from 
different populations, or different intervention strategies were used. The review included clinical trials published up to $2011^{38}$.

Children with severe acute malnutrition who also have diarrhea and are dehydrated need special alternative fluids to treat dehydration. The current recommendations for the management of diarrheal disease, are based on WHO guidelines.

- The management of gastroenteritis in malnourished children is different from that recommended in well-nourished children and it is broad and highly variable according to a recent survey by FISPGHAN working group (Lo Vecchio A et al, manuscript in preparation) and there is a need for specific guidelines.

The recommendations for the management of gastroenteritis need to be regularly updated in the light of new evidence available. They should be based on universal principles, but tailored to geographical, epidemiological, and organizational conditions.

Finally, there is non-conclusive evidence supporting a relationship between early AGE and subsequent functional disorders later in life ${ }^{39}$. The cause-effect relationship is unknown and deserves attention.

\section{Recommendations by the Working Group and action points}

Reduction in mortality rates from diarrhea in Sub-Saharan countries and South Asia is still far from satisfactory. More resources should be directed at maternal health care services and educational programs for mothers and adolescents and at improving access to safe drinking water and sanitation ${ }^{7}$ as indicated in the previous FISPGHAN report ${ }^{2}$. In addition to immunization, AGE might be prevented much more effectively by socio-economic, educational, and hygienic infrastructural interventions. Physicians and scientific societies should play a proactive role in lobbying, promoting education and through other initiatives. FISPGHAN produced a video targeting mothers and field workers giving simple guidance in 
form of cartoons for early recognizing dehydration https://www.youtube.com/watch?v=IYW9hpcAKwk). Those initiatives should be conducted on a large scale using the available quad-media and mass communication, including social media. Many questions on diarrhea management require interventions and active research.

\section{Recommendations needed}

- Management of children treated at hospital because of AGE (this includes management in the emergency area and the inpatient facility)

- Specific management of children belonging to high risk groups, including malnourished children, those with chronic conditions, and neonates

- Evidence-based indications for anti-infective therapy

- Optimal composition of oral rehydration solution for well-nourished and malnourished children

\section{Research issues}

- Under what conditions is the detection of a diarrhea-associated microbe a diarrheal pathogen? Importance of qualitative, quantitative, and patient-based diagnostic approaches

- Stunting as a risk factor for diarrheal deaths

- Environmental enteric dysfunction as a diarrhea-independent process and its negative impact on the course of AGE

- First 1000 days (gut infections during pregnancy, lactation, and early childhood) as a risk for diarrhea and a target for prevention

- Efficacy and safety of specific drugs, antibiotics, probiotics, and diet to reduce the duration and intensity of diarrhea 
- Efficacy of RV vaccine in different geographical settings

- Cause-effect relationships between intestinal infections and subsequent intestinal functional disorders

- Microbiome-directed refeeding during and following diarrheal episodes

- High quality randomized controlled trials on drugs or other sustainable interventions that effectively reduce duration and intensity of AGE

\section{Future priorities of the Working Group}

- Implementation of the universal guidelines for management of gastroenteritis based on evolving evidence, local resources and data.

- Advocacy for research funding and implementation science for current best practices, informed by pragmaticism and resources

- New vaccines for high burden pathogens

- Broader dissemination of WHO recommendation for the treatment, prevention, and effective support to children who live in difficult conditions

\section{DISCLAIMER:}

"FISPGHAN is not responsible for the practices of physicians and provides position papers as indicators of best practice only. Diagnosis and treatment are at the discretion of physicians". 


\section{References:}

1. GBD 2016 Diarrhoeal Disease Collaborators, et al. Estimates of the global, regional, and national morbidity, mortality, and aetiologies of diarrhoea in 195 countries. Lancet Infect Dis. 2018;18:1211-28.

2. Guarino A, Winter H, Sandhu B, et al. Acute gastroenteritis disease: Report of the FISPGHAN Working Group. J Pediatr Gastroenterol Nutr. 2012;55:621-6.

3. Lo Vecchio A, Dias JA, Berkley JA et al. Comparison of recommendations in clinical practice guidelines for acute gastroenteritis in children. J Pediatr Gastroenterol Nutr. 2016;63:226-35.

4. Guarino A, Lo Vecchio A, Dias JA, et al. Universal recommendations for the management of acute diarrhea in non-malnourished children. $J$ Pediatr Gastroenterol Nutr. 2018; 67:586-93.

5. Nicastro, E, Lo Vecchio A, Liguoro I, et al. The impact of e-learning on adherence to guidelines for acute gastroenteritis: A single-arm intervention study. PLoS One. 2015;10:e0132213.

6. Liu L, Za S, Hogan D, et al. Global, regional, and national causes of under-5 mortality in 2000-15: an updated systematic analysis with implications for the Sustainable Development Goals. Lancet. 2016; 388:3027-35.

7. Acheampong M., Ejiofor C, Salinas-Miranda A. An analysis of determinants of under-5 mortality across countries: defining priorities to achieve targets in sustainable developmental goals. Matern Child Health J. 2017; 21:1428-47.

8. Thongprachum A, Takanashi S, Kalesaran AF, et al. Four-year study of viruses that cause diarrhea in Japanese pediatric outpatients. J Med Virol. 2015;87:1141-8.

9. Kotloff KL, Nataro JP, Blackwelder WC, et al. Burden and aetiology of diarrhoeal disease in infants and young children in developing countries (the Global Enteric 
Multicenter Study, GEMS): a prospective, case-control study. Lancet 2013;382: 209-22.

10. Operario D. J, Platts-Mills JA, Nadan S, et al. Etiology of severe acute watery diarrhea in children in the global Rotavirus surveillance network using quantitative polymerase chain reaction. J Infect Dis. 2017; 216:220-7.

11. Liu J, Platts-Mills JA, Juma J, et al. Use of quantitative molecular diagnostic methods to identify causes of diarrhoea in children: a reanalysis of the GEMS casecontrol study. Lancet 2016;388:1291-301.

12. Platts-Mills JA, Liu J, Rogawski ET, et al. Use of quantitative molecular diagnostic methods to assess the aetiology, burden, and clinical characteristics of diarrhoea in children in low-resource settings: a reanalysis of the MAL-ED cohort study. Lancet Glob Health. 2018;6: e1309-18.

13. Guarino A, Alessio M, Tarallo L, et al. Heat stable enterotoxin produced by Escherichia coli in acute diarrhoea. Arch. Dis. Child. 1989; 64:808-13.

14. Anderson, NW, Tarr PI. Multiplex nucleic acid amplification testing to diagnose gut infections: challenges, opportunities, and result interpretation. Gastroenterol Clin North Am. 2018;47:793-812.

15. Sinclair D, Abba K, Zaman K, et al. Oral vaccines for preventing cholera. Cochrane Database Syst Rev. 2011;(3):CD008603.

16. Milligan R, Paul M, Richardson M, et al. Vaccines for preventing typhoid fever. Cochrane Database Syst Rev. 2018; 5:CD001261.

17. Soares-Weiser K, Bergman H, Henschke N, et al. Vaccines for preventing rotavirus diarrhoea: vaccines in use. Cochrane Database Syst Rev. 2019;(10):CD008521.

18. Clark, A., van Zandvoort K, Flasche S, et al. Efficacy of live oral rotavirus vaccines by duration of follow-up: a meta-regression of randomised controlled trials. Lancet Infect Dis. 2019;19:717-27. 
19. Vesikari T, Van Damme P, Giaquinto C, et al. European Society for paediatric infectious diseases Consensus Recommendations for Rotavirus Vaccination in Europe: Update 2014. Pediatr. Infect. Dis. J. 2015;34:635-43.

20. Lo Vecchio A, Liguoro I, Dias JA et al. Rotavirus immunization: Global coverage and local barriers for implementation. Vaccine. 2017;35:1637-44.

21. Harro C, Louis Bourgeois A, Sack D. et al. Live attenuated enterotoxigenic Escherichia coli (ETEC) vaccine with dmLT adjuvant protects human volunteers against virulent experimental ETEC challenge. Vaccine 2019;37:1978-86.

22. Falszewska A, Szajewska H, Dziechciarz P. Diagnostic accuracy of three clinical dehydration scales: a systematic review. Arch Dis Child. 2018;103:383-8.

23. Anigilaje EA. Management of diarrhoeal dehydration in childhood: A review for clinicians in developing countries. Frontiers Pediatr 2018;6:28

24. Rutman L, Klein EJ, Brown JC. Clinical Pathway Produces Sustained Improvement in Acute Gastroenteritis Care. Pediatrics. 2017;140(4). pii:e20164310.

25. Rouhani S, Meloney L, Ahn R, et al. Alternative rehydration methods: A systematic review and lessons for resource-limited care. Pediatrics 2011;127:e748- 57.

26. Giannattasio A, Guarino A, Lo Vecchio A. Management of children with prolonged diarrhea [version 1; peer review: 3 approved]. F1000Research 2016, 5(F1000 Faculty Rev):206 (https://doi.org/10.12688/f1000research.7469.1)

27. Leibovici-Weissman, Y, Neuberger A, Bitterman R, et al. Antimicrobial drugs for treating cholera. Cochrane Database Syst Rev. 2014 Jun 19:(6)CD008625.

28. Tickell, K. D, Brander RL, Atlas HE et al. Identification and management of Shigella infection in children with diarrhoea: a systematic review and metaanalysis. Lancet Glob Health. 2017; 5: e1235-48. 
29. Giannattasio A, Guarino A, Lo Vecchio A. Management of children with prolonged diarrhea [version 1; peer review: 3 approved]. F1000Research 2016, 5(F1000 Faculty Rev):206 (https://doi.org/10.12688/f1000research.7469.1)

30. Wen SCH, Best E, Nourse C. Non-typhoidal Salmonella infections in children: Review of literature and recommendations for management. J Paediatr Child Health 2017;53: 936-41.

31. Guarino, A. Ashkenazi S, Gendrel D, et al. European society for pediatric gastroenterology, hepatology, and nutrition/European society for pediatric infectious diseases evidence-based guidelines for the management of acute gastroenteritis in children in Europe: Update 2014. J Pediatr Gastroenterol Nutr 2014; 59:132-52 .

32. Williams PCM, Berkley JA. Guidelines for the treatment of severe acute malnutrition: a systematic review of the evidence for antimicrobial therapy. Paediatr Int Child Health. 2018;38:S32-S49. 
33. MacGillivray S., Fahey T, McGuire W. Lactose avoidance for young children with acute diarrhoea. Cochrane Database Syst Rev. 2013;(10):CD005433.

34. Bandsma RHJ, Voskuijl W, Chimwezi E, et al. A reduced- carbohydrate and lactose-free formulation for stabilization among hospitalized children with severe acute malnutrition: A double-blind, randomized controlled trial. PLoS Med. 2019; 16:e1002747.

35. Lo Vecchio A, Vandenplas Y, Benninga M, et al. An international consensus report on a new algorithm for the management of infant diarrhoea. Acta Paediatr. 2016; 105, e $384-9$.

36. The top 10 causes of death. Available at: https://www.who.int/news-room/factsheets/detail/the-top-10-causes-of-death. (Accessed: 30th August 2019)

37. WHO | World Health Statistics 2018: Monitoring health for the SDGs. WHO. 2018.

38. WHO. Guideline: Updates on the management of severe acute malnutrition in infants and children. Geneva: World Health Organization. 2013.

39. Cocciolillo S, Collins SM. The long-term functional consequences of acute infectious diarrhea. Curr Opin Gastroenterol. 2016;32:1-6. 
Fgure 1. Barriers to immunization against Rotavirus

Although the single major barrier is "cost", underscoring the severity of AGE by caregivers, health authorities, and even by physician is also a major barrier. Hence, knowledge rather than cost is a target of actions to ensure widespread universal immunization. Organizational issues are an additional barrier, very much related to the need of keeping the vaccine viable under temperature control and the need of administering the vaccine within a narrow time window early after birth. From ref 20, modified

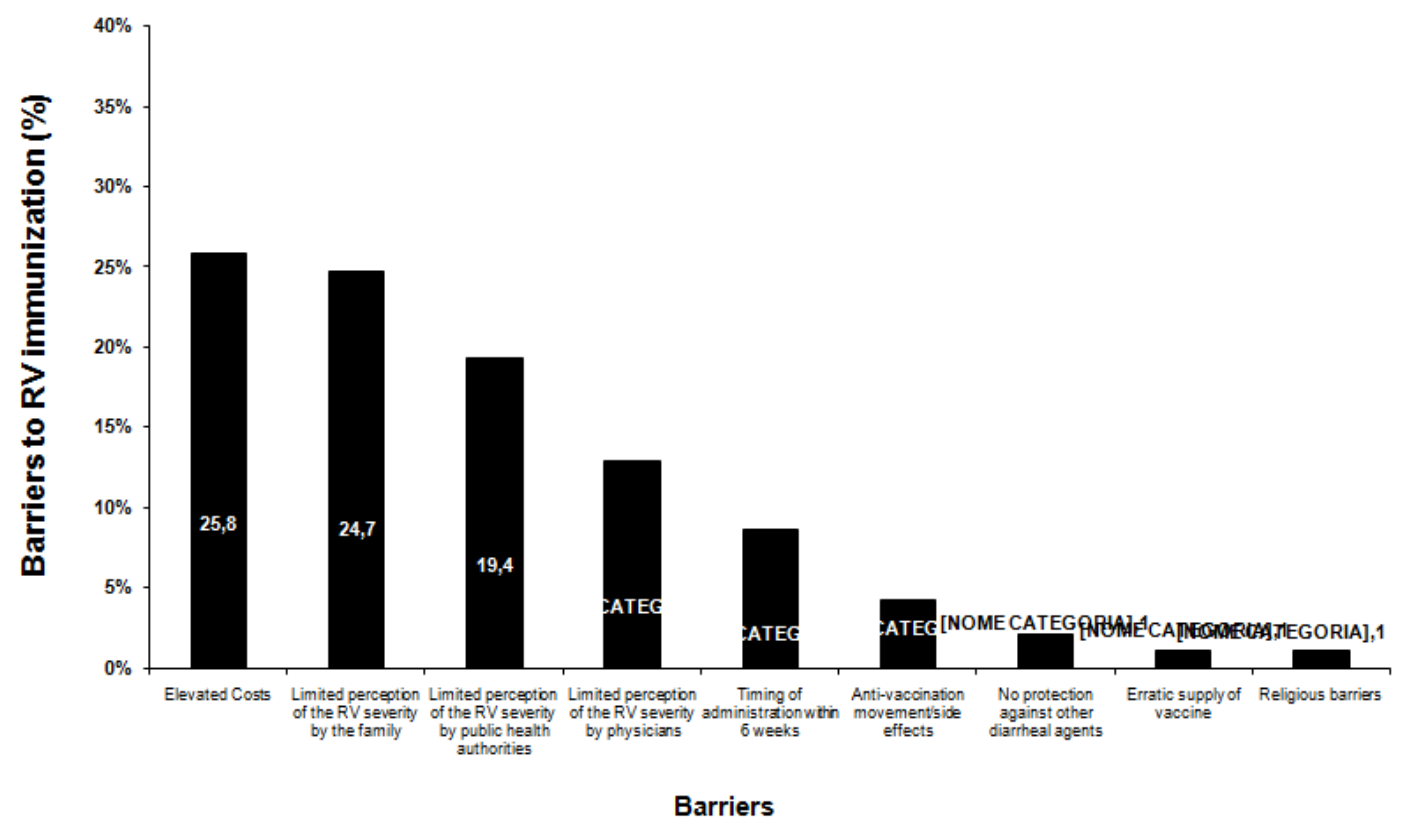


Figure 2: Algorithm for the management of AGE. The algorithm shows indications providing clear information on what to do in the presence of red flags. Prolonged diarrhea is considered as a complication requiring investigations. Oral rehydration solution should always be regarded as the key intervention to start with in all cases. From ref 35, modified

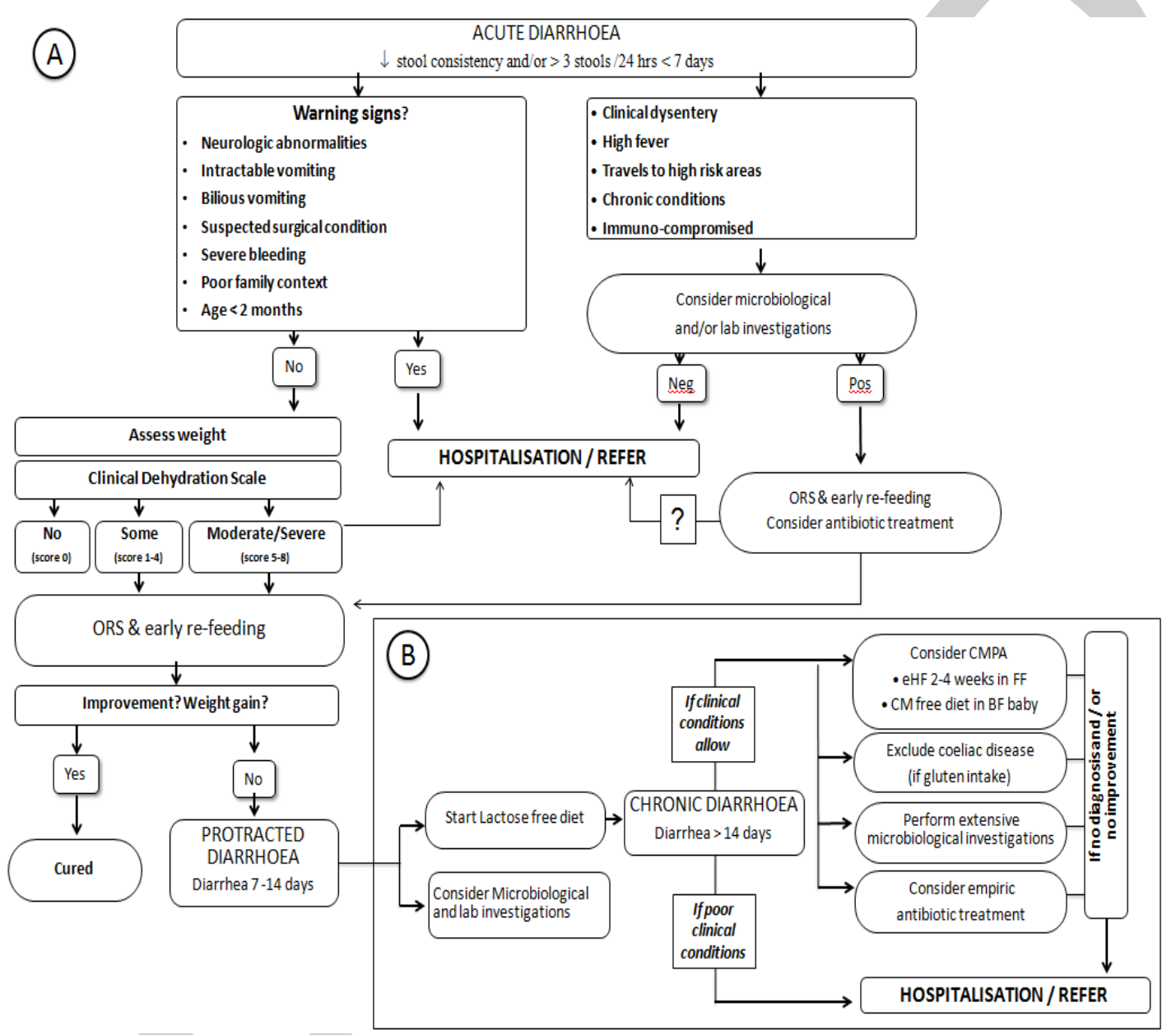


Table 1: Priorities indicated by the FISPGHAN Working Group report 2012 (from ref 2)

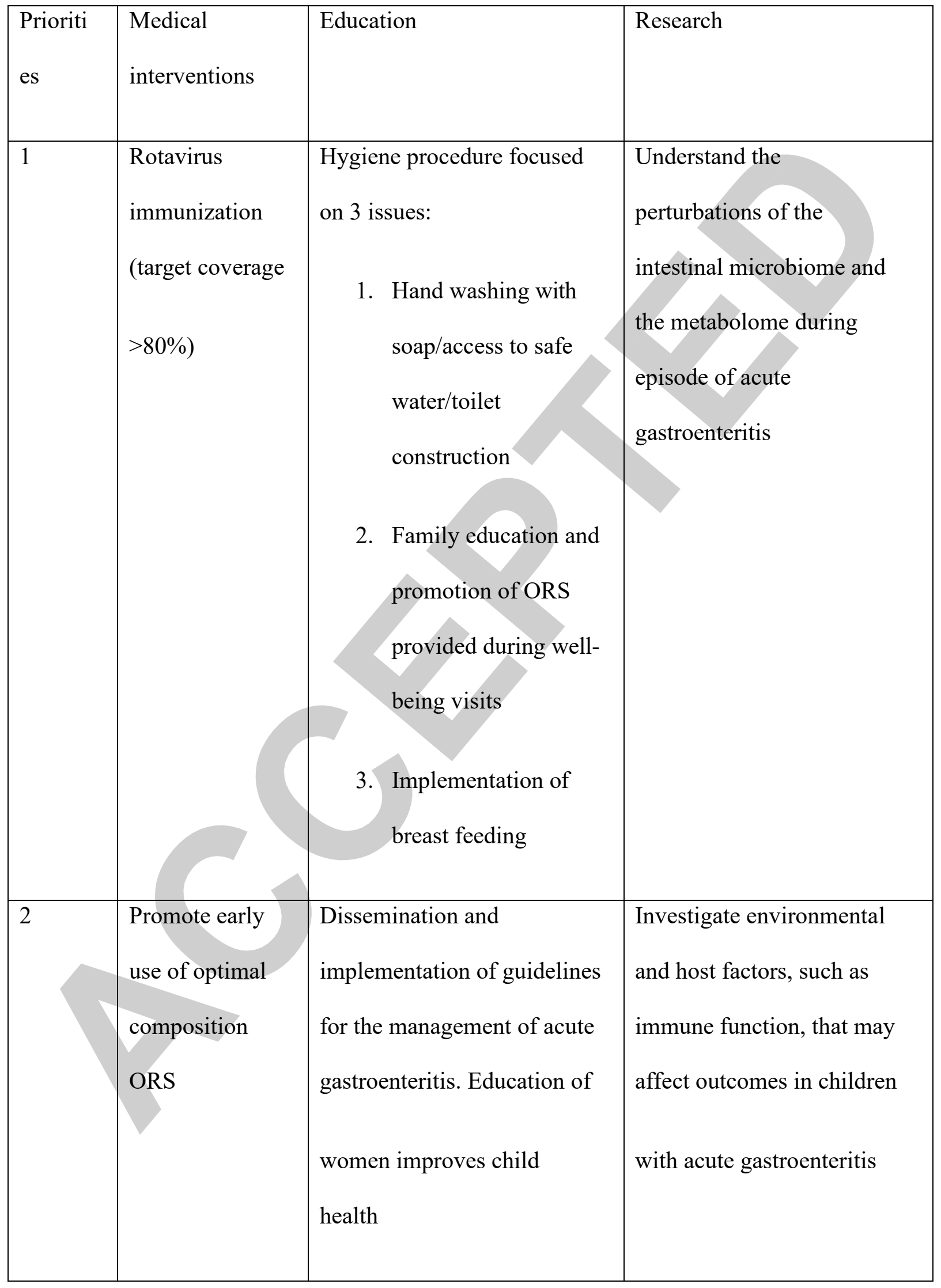




\begin{tabular}{|c|c|c|c|}
\hline 3 & $\begin{array}{l}\text { Reduce } \\
\text { inappropriate } \\
\text { medical } \\
\text { interventions }\end{array}$ & $\begin{array}{l}\text { e-Learning programs for a } \\
\text { worldwide education on } \\
\text { acute gastroenteritis with an } \\
\text { emphasis on education of } \\
\text { girls and women }\end{array}$ & $\begin{array}{l}\text { Apply systems biology } \\
\text { methodology integrating } \\
\text { host, microbial, } \\
\text { immunologic, genetic, and } \\
\text { epigenetic factors to identify } \\
\text { novel metabolic pathways, } \\
\text { which will lead to the } \\
\text { discovery of new } \\
\text { therapeutic interventions } \\
\text { and more } \\
\text { effective ORS. }\end{array}$ \\
\hline
\end{tabular}


Table 2: Prevention rates from case-controlled strains for RV vaccine in high-income and lowincome countries in the first and second year of vaccination*

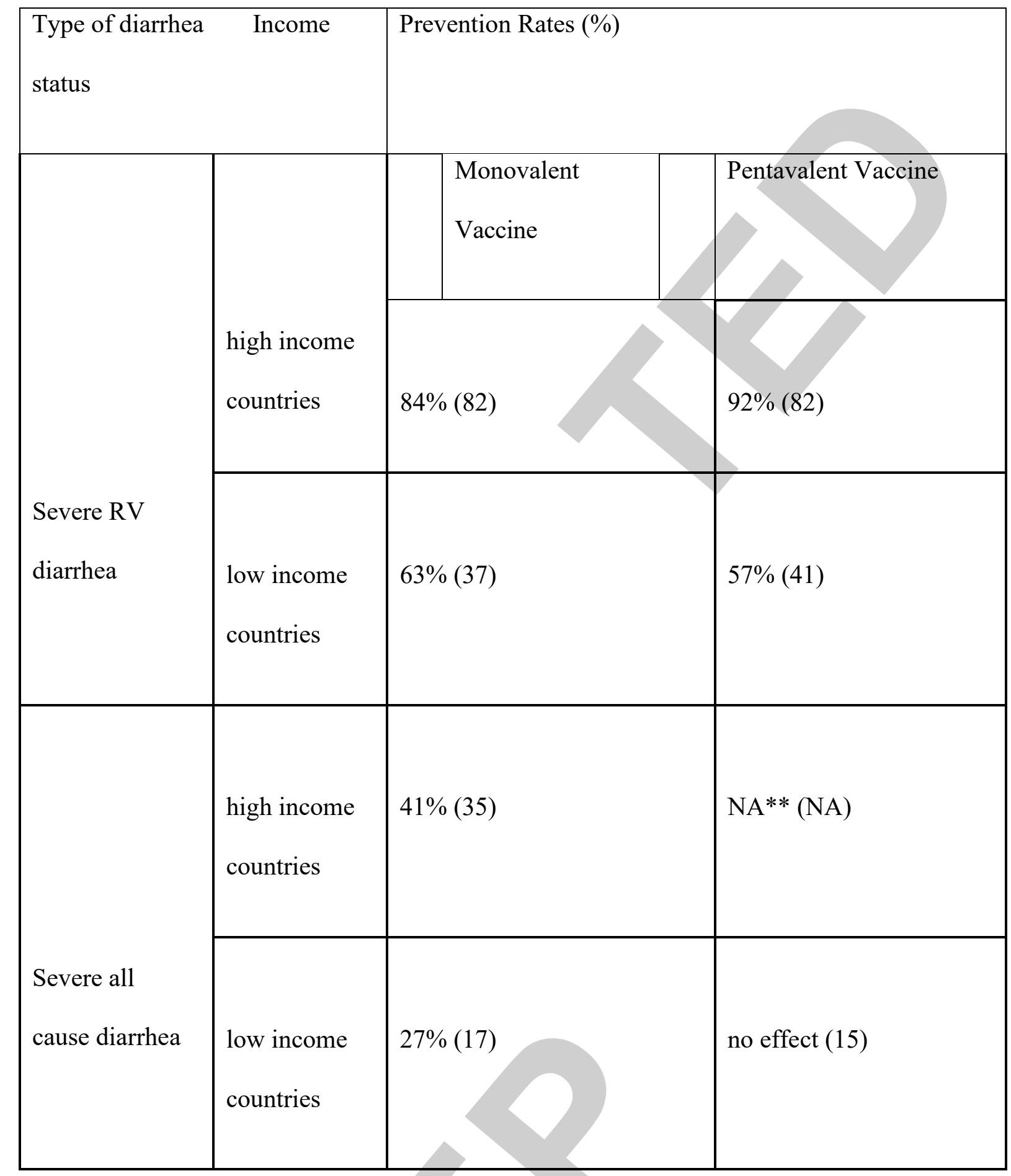

* Based on data from ref 17

$* * \mathrm{NA}=$ not available 


\section{Table 3: Potential indications for antibiotic therapy in AGE (A) and antibiotics choice (B)}

A.

\begin{tabular}{|l|l|l|}
\hline Clinical condition & Host-related factors & Setting-related risk factors \\
\hline Dysenteric diarrhea & Age $<3$ (or 6) months & Infants day care settings \\
inflammation markers & Invasive bacterial infections & Hospitals \\
\hline Prolonged diarrhea & Severe acute malnutrition & Closed institutions \\
\hline Small intestinal bacterial & Chronic underlying disease & Traveler's diarrhea \\
\hline overgrowth & & \\
\hline Antibiotic-associated & Immunodeficiency & \\
diarrhea & & \\
\hline Toxic state & & \\
\end{tabular}

Table 3: Potential indications for antibiotic therapy in AGE (A) and antibiotics choice (B)

B. 


\begin{tabular}{|c|c|c|}
\hline Condition & First finding & Alternative drugs \\
\hline Small intestine & Cotrimoxazole & \\
\hline Bacterial overgrowth & Metronidazole & \\
\hline Invasive/Toxic state & Ceftriaxone & Ampicillin \\
\hline Antibiotics & Metronidazole & \\
\hline Campylobacter jejuni & Azithromycin & Ciprofloxacin, vancomycin \\
\hline Clostridium difficile & Metronidazole & Vancomycin \\
\hline Non -typhoidal & Amoxicillin or ceftriaxone & $\begin{array}{l}\text { Trimethoprim- } \\
\text { sulfamethoxazole }\end{array}$ \\
\hline Salmonella typhi & $\begin{array}{l}\text { Third-generation } \\
\text { cephalosporins }\end{array}$ & Chloramphenicol \\
\hline Shigella & Azithromycin, ceftriaxone & Cefixime, ciprofloxacin \\
\hline Yersinia & $\begin{array}{l}\text { Trimethoprim } \\
\text { sulfamethoxazole }\end{array}$ & Ceftriaxone \\
\hline Vibrio cholerae & Azithromycin & $\begin{array}{l}\text { Doxycycline ( }<8 \text { years }) \text {, } \\
\text { ciprofloxacin }\end{array}$ \\
\hline ETEC & $\begin{array}{l}\text { Azithromycin (only for } \\
\text { traveler's diarrhea) }\end{array}$ & $\begin{array}{l}\text { Trimethoprim } \\
\text { sulfamethoxazole }\end{array}$ \\
\hline
\end{tabular}


ETEC, enterotoxigenic Escherichia coli 
Table 4: The ideal antidiarrheal drug

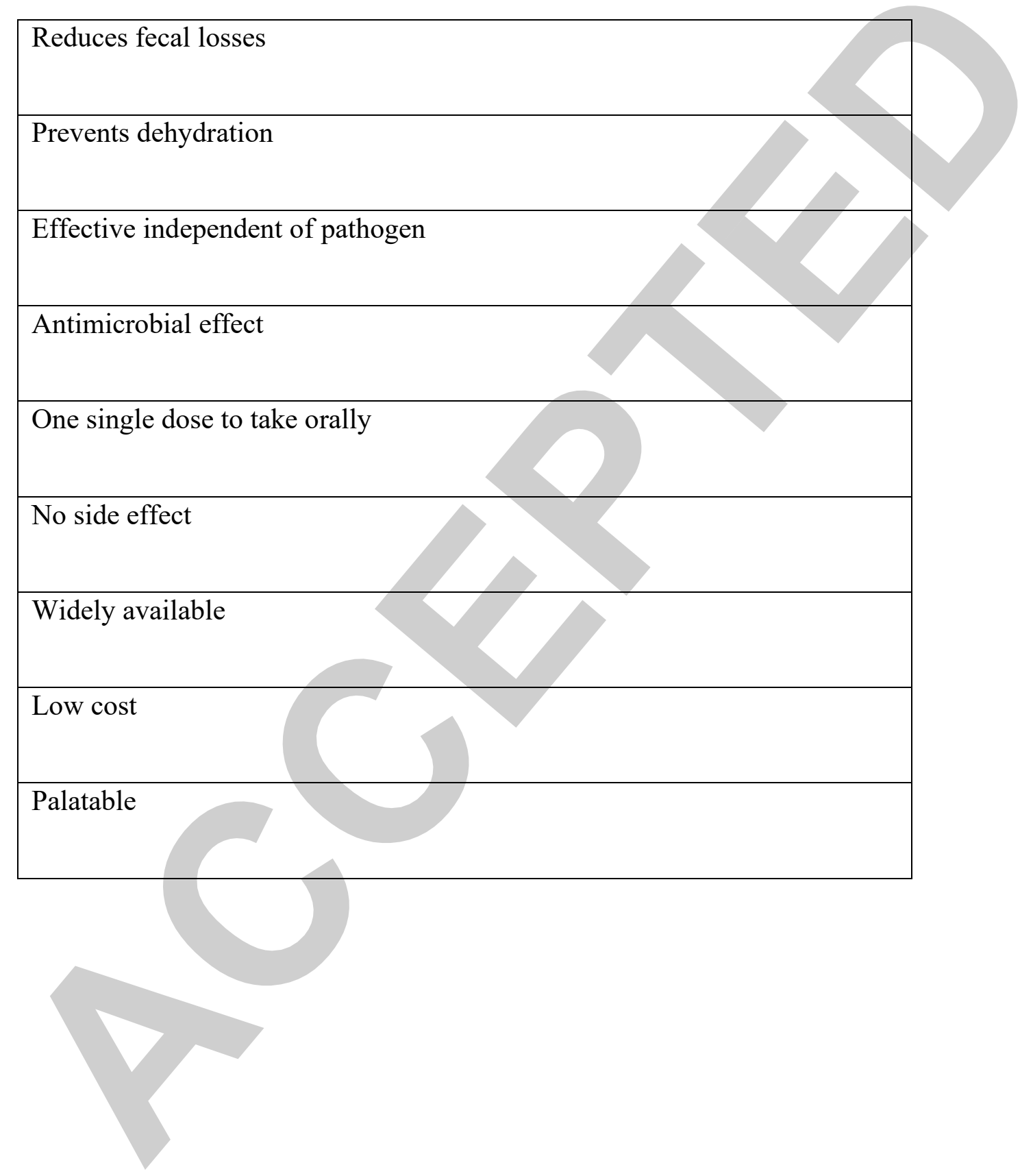

